\title{
Prevalência do não uso de preservativo entre universitários e pós- graduandos de uma universidade pública do Norte do Brasil
}

\author{
Prevalence of no condom use among undergraduates and post-graduates of a public \\ university in Northern Brazil
}
Prevalencia de no usar condón preservative entre universitarios y postgraduados de una universidad publica del Norte de Brasil

Jeanne Lúcia Gadelha Freitas ${ }^{1 *}$, Priscilla Perez da Silva Pereira ${ }^{1}$, Kátia Fernanda Alves Moreira ${ }^{1}$, Adriana Dias Silva ${ }^{1}$.

\section{RESUMO}

Objetivo: Identificar a prevalência do não uso do preservativo nas últimas cinco relações sexuais entre universitários de uma instituição pública do Norte do Brasil. Métodos: Estudo transversal, com 772 estudantes do ensino superior e pós-graduandos. Foi utilizando um questionário fechado com respostas autorreferidas. A razão de prevalência das variáveis foi analisada pela regressão de Poisson. Resultados: A maioria dos participantes são adultos jovens, sexualmente ativos, com parceiro único e utilizaram o preservativo em pelo menos uma vez das últimas cinco relações sexuais. O não uso do preservativo teve maior prevalência entre estudantes com parceiro único, acima de 40 anos e cursando os últimos períodos dos cursos. Conclusões: Os universitários com um único parceiro sexual em períodos finais de curso estão mais vulneráveis às Infecções Sexualmente transmissíveis pelo uso inconsistente do preservativo. A universidade pode ser um ambiente propicio para ações de promoção à saúde sexual e reprodutiva que visem alcançar, sobretudo os estudantes com parceiro único e em vias de conclusão do curso.

Palavras-chave: Estudantes, Preservativos, Prevalência.

\begin{abstract}
Objective: To identify a prevalence of no condom use in the last five sexual relations between university students of the public institution of the North of Brazil. Methods: Cross-sectional study with 772 undergraduate students and postgraduate students. It was using a closed questionnaire with self-reported answers. The prevalence ratio of the variables was analyzed by Poisson regression. Results: Most of the participants are young, sexually active, single-partner adults who have used the condom at least once in the past five sexual encounters. The non-use of condoms was more prevalent among students with a single partner, over 40 years of age and attending the last periods of the courses. Conclusions: College students with a single sexual partner in final periods of the course are more vulnerable to Sexually Transmitted Infections due to the inconsistent use of condoms. The university can be an environment conducive to actions to promote sexual and reproductive health, which reach, especially students with a single partner and in the process of completing the course.
\end{abstract}

Keywords: Students, Condoms, Prevalence.

${ }^{1}$ Universidade Federal de Rondônia (UNIR), Porto Velho-Rondônia *E-mail: jeannegadelha@unir.br

SUBMETIDO EM: 4/2019

ACEITO EM: 5/2019

PUBLICADO EM: 7/2019 


\section{RESUMEN}

Objetivo: Identificar la prevalencia del no uso del condón en las últimas cinco relaciones sexuales entre universitarios de una institución pública del Norte de Brasil. Métodos: Estudio transversal, con 772 estudiantes de enseñanza superior y postgraduados. Se utilizó un cuestionario cerrado con respuestas autorreferidas. La razón de prevalencia de las variables fue analizada por la regresión de Poisson.

Resultados: La mayoría de los participantes son adultos jóvenes, sexualmente activos, con un socio único y utilizaron el condón en al menos una vez de las últimas cinco relaciones sexuales. El no uso del preservativo tuvo mayor prevalencia entre estudiantes con pareja única, por encima de 40 años y cursando los últimos períodos de los cursos. Conclusiones: Los universitarios con un único socio sexual en períodos finales de curso son más vulnerables a las Infecciones Sexualmente transmisibles por el uso inconsistente del preservativo. La universidad puede ser un ambiente propicio para acciones de promoción a la salud sexual y reproductiva que tengan como objetivo alcanzar, sobre todo los estudiantes con pareja única y en vías de conclusión del curso.

Palabras clave: Estudiantes, condones, Prevalencia.

\section{INTRODUÇÃO}

Entre os principais agravos à saúde sexual e reprodutiva de jovens estão as Infecções Sexualmente Transmissíveis (IST) incluindo o Vírus da Imunodeficiência Humana (HIV), responsável pela síndrome da imunodeficiência Adquirida (SIDA), a gravidez não planejada e a violência, imbricadas nas relações de gênero (ARRIAGADA BEM e BERTONI JS, 2014; PEREIRA BS et al., 2014).

Os jovens adultos, dentre os os universitários, constituem um grupo vulnerável para os agravos relacionados ao comportamento sexual de risco como trocas de parceiro frequente, baixa adesão ao uso do preservativo, uso frequente de álcool e outras substâncias psicoativas no momento do ato sexual por comprometer a percepção de vulnerabilidade pessoal (PEREIRA BS et al., 2014; Bezerra EO et al., 2012).

No Brasil, a prevenção das IST/HIV/Aids é um desafio em todas as faixas etárias, mas, sobretudo entre os jovens. Em 2015, quase metade dos 8.027.297 milhões de universitários tinham entre 18 a 24 anos, coincidindo com a faixa etária com maior incidência de novos casos de Aids do país (BRASIL, 2015).

Nos últimos dez anos, mais da metade das novas infecções pelo HIV ocorreram entre adolescentes e adultos jovens de até 34 anos. Do total de casos de Aids nesse período, 8,7\% eram universitários no momento do diagnóstico e 12,5\% haviam concluído a graduação (BRASIL, 2018).

A chegada dos jovens à vida adulta, geralmente coincide com o ingresso na universidade, provocando mudanças nas atitudes e comportamento de risco à saúde em vários aspectos (ARRIAGADA BEM e BERTONI JS, 2014; GRAVATA A et al., 2016).

Para muitos destes jovens, à medida que assumem maiores responsabilidades, autonomia financeira e poder de decisão, passam também a ter maior contato e oportunidades de experimentar álcool, drogas e a prática de sexo inseguro caracterizado pelo não uso do preservativo, tornando-os mais vulneráveis às IST/HIV/Aids e a gravidez não desejada (SALES WB et al., 2016).

Em alguns países, incluindo o Brasil, o uso do preservativo entre jovens foi popularizado em todas as camadas sociais e tem sido um fator muito importante no controle da epidemia de HIV/Aids (VALENCIA CP e CARVAJAL, EG, 2012; REYNOLDS HW et al., 2012).

Mas a despeito dos avanços tecnológicos, da liberdade de escolhas e livre acesso às informações, alguns estudantes ainda apresentam um comportamento de vulnerabilidade sexual (JANEIRO JM et al., 2013; DOURADO I et al., 2015). Os motivos vão desde o desconhecimento sobre o tema, a crença de que não terá riscos de se contaminar, uso do preservativo nos seus relacionamentos sexuais, a dificuldade de acesso e até a decisão de transgredir regras sociais vigentes (SALES WB et al., 2016, El BCHERAOUI et al., 2013). 
Por outro lado, o ambiente universitário favorece a construção de novos saberes e a interação com diversos indivíduos, predispondo os estudantes às mudanças de atitude e conduta sexual saudável (FIRMEZA SNRM et al., 2016). Um estudo sobre autopercepção e o desconhecimento sobre as IST entre acadêmicos veteranos e calouros do sudoeste do país, mostrou que mais de $70 \%$ dos acadêmicos, incluindo os da graduação em Medicina, reconheceram ter dúvida sobre o tema, se dispuseram a ler os comentários e afirmaram que pretendiam ler mais sobre as IST após terem participado da pesquisa. Entre que foram até o final do estudo, mais de $95 \%$ aprenderam algo sobre as infecções relacionadas ao exercício da sexualidade (CASTRO EL et al., 2016).

Estas evidências coadunam com a preocupação de profissionais de saúde nos diferentes níveis de atenção à saúde da população de jovens e adultos, considerando que a atividade sexual iniciada na adolescência se estende para vida adulta e com essa iniciação também uma maior vulnerabilidade às IST, a gestação não planejada e aborto (DOURADO I et al., 2015). Assim, o uso de métodos contraceptivos, sobretudo do preservativo, é desejável e constitui-se em um dos marcos da vivência saudável da sexualidade na adolescência e vida adulta (FIRMEZA SNRM et al., 2016).

Um estudo sobre o início da vida sexual e a contracepção de 74.589 adolescentes e jovens brasileiros revelou que a média de idade da primeira relação sexual foi de 17 anos. Entretanto, a idade da iniciação sexual não teve distribuição homogênea no país e esteve ligada a fatores como local de residência, tipo de escola, crenças religiosas e culturais. Quanto ao uso de preservativos, na região Norte, 69,4\% dos participantes referiu uso de preservativo na última relação sexual (BORGES ALV et al., 2017).

Com vistas à evolução da epidemia de Aids no Brasil, este indicadores se mostram significativos quando se constata também que na região Norte do país, houve um crescimento linear de casos de 15,0\% para $24,0 \%$ nos últimos dez anos, sinalizando importantes desafios para politicas de prevenção às IST/HIV/aids nesta região, incluindo os jovens ingressantes na universidade, formadores de opiniões como futuros profissionais (BRASIL, 2016).

Considerando o exposto e a problemática do pouco conhecimento sobre a prevalência de uso do preservativo entre adultos jovens, o objetivo desta investigação foi identificar a prevalência de fatores relacionados ao não uso do preservativo nas últimas cinco relações sexuais entre universitários e pósgraduandos de uma universidade pública no norte do Brasil.

\section{MÉTODOS}

Trata-se de uma pesquisa do tipo transversal realizado na Universidade Federal de Rondônia no campus José Ribeiro Filho, na capital de Porto Velho. Nesse campus, no ano de 2015, estavam matriculados 3.931 estudantes, distribuídos em 29 cursos de graduação e 16 programas de pós-graduação. Desses, os 17 cursos de graduação presencial e dois de pós-graduação, com horário de aulas no período matutino e vespertino, foram objetos da pesquisa.

Foram entrevistados 1.076 estudantes extraídos de uma composição amostral não probabilística e obtida por conveniência, pelo consentimento voluntário dos participantes. Considerou-se como critérios de inclusão, universitários e pós-graduandos que estivessem presentes na sala de aula no dia da coleta de dados. Foram excluídos os estudantes que nunca tiveram relações sexuais e que preencheram parcialmente o questionário da pesquisa.

A coleta de dados ocorreu no período de março a novembro de 2016, uma vez por semana em dias alternados e em diferentes horários. Foi utilizando questionário estruturado, com vinte questões fechadas, de autopreenchimento e anônimo. $O$ instrumento de pesquisa utilizado foi um questionário do tipo CAP (Conhecimento, Atitude e Prática) proveniente de uma pesquisa matriz intitulada "Conhecimento sobre DST/HIV/Aids e outros agravos: Atitudes e práticas preventivas entre Universitários", desenvolvido por meio de projeto de extensão institucional. Os questionários foram do tipo autoaplicável pelos participantes em sala de aula, após orientações gerais dos pesquisadores previamente treinados. 
A variável dependente foi o não uso do preservativo nas últimas cinco relações sexuais. Considerou-se que o uso apenas na última relação poderia levar a um resultado que não refletisse o real comportamento dos participantes. Ao considerar o uso nas últimas cinco relações pode ser inferido um comportamento e não apenas uma ação isolada, ocorrida pelo acaso. As variáveis independentes abordaram aspectos sociodemográficos, educacionais e do comportamento sexuais assim categorizadas:

- Aspectos sociodemográficos: Idade (16 a 20 anos; 21 a 25 anos; 26 a 31 anos; 31 a 35 anos; 36 a 40 anos; $\geq 41$ anos); sexo (masculino ou feminino); raça/cor da pele (branco ou não branco autodeclarado como pardo, negro, indígena ou asiático); estado civil (com companheiro que se refere à união estável ou casado, e sem companheiro que se refere a solteiro, namorando, viúvo e divorciado); classe/renda familiar (menos de dois salários mínimos, 2 a 4 salários ou superior a 5 salários mínimos ou mais); religião (protestantes, católicos, sem religião ou outras religiões: Budismo, Judaísmo, Santo Daime, Umbanda, Candomblé e União Vegetal).

- Aspectos educacionais: curso matriculado (curso da área da saúde ou curso que não é da área da saúde), período do curso (iniciante: $1^{\circ}$ ao $3^{\circ}$ semestre; intermediário: $4^{\circ}$ ao $6^{\circ}$ semestre; término: $7^{\circ}$ ao $10^{\circ}$ semestre ou pós-graduação), fonte de informação em saúde preferida (pessoal: informação advinda de professores, amigos, familiares, profissionais da área de saúde, na igreja e no trabalho versus informações de fonte não pessoais advindas de artigos científicos, folhetos, panfletos, livros, jornais, internet, televisão e rádio).

- Aspectos do comportamento sexual: número de parceiros sexuais nos últimos 12 meses (nenhum; um parceiro; dois; três ou mais parceiros).

$\mathrm{Na}$ análise de dados realizou-se a análise estatística com cálculos de frequência absoluta $(\mathrm{n})$ e relativa (\%), média e desvio padrão (DP). Investigaram-se a Razão de Prevalência (RP) e seus respectivos Intervalos de Confiança de $95 \%$ como medida de efeito obtida pela análise de regressão de Poisson entre o não uso do preservativo e as variáveis independentes por meio de análises bivariadas.

Todas as variáveis com significância $p<0,20$ na análise bivariada foram incluídas no modelo hierárquico final, onde o bloco com variáveis sociodemográficas (1) é o mais distal e o bloco comportamento sexual (3) mais proximal para desfecho de estudo. Obteve-se três modelos de regressão, onde as Razões de Prevalência foram ajustadas pelas variáveis do mesmo nível e variáveis do bloco anterior. O teste de HosmerLemeshow foi utilizado para verificar a qualidade dos ajustes dos modelos empregados. Os dados foram processados no software Stata ${ }^{\circledR}$ (College Station, Texas, USA) versão 13.

Atenderam-se os preceitos éticos referentes à condução de estudos envolvendo seres humanos de acordo com a Declaração de Helsinque e a Resolução CNS no 466, de 12 de dezembro de 2012, do Conselho Nacional de Saúde. Esta pesquisa foi aprovada pelo Comitê de Ética em Pesquisa da Universidade Federal de Rondônia sob o número FR439664.

\section{RESULTADOS}

Dos 1.076 estudantes universitários e pós-graduando entrevistados, 97,2\% já haviam tido a primeira relação sexual e destes, 772 responderam todas as perguntas do questionário sendo assim incluídos nesta investigação. Portanto, a proporção de resposta foi de 74,0\%.

Entre os 772 participantes, 40,6\% utilizaram preservativo em todas as últimas cinco relações sexuais, $24,4 \%$ não usaram preservativo nenhuma vez e $35 \%$ usaram o preservativo em pelo menos uma vez nas últimas cinco relações.

A Tabela 1 apresenta as características sociodemográficas dos participantes. A maioria dos participantes tinha menos de 25 anos (56,7\%) sendo que a idade média dos estudantes foi de 27,1 ( $D P=8,63)$. Metade dos participantes era do sexo feminino (50,5\%). A maioria se declarava não branco $(71,8 \%)$, sem companheiro, ou seja, não tinham união estável ou casados $(65,5 \%)$ e tinham renda familiar de até quatro salários mínimos por família $(62,6 \%)$. Entre as religiões o catolicismo foi a mais predominante $(40,2 \%)$. 
Tabela 1 - Características sociodemográficas dos universitários e pós-graduandos e não uso de preservativo nas últimas cinco relações sexuais, $\mathrm{n}=772$. Porto Velho, RO, 2016.

\begin{tabular}{|c|c|c|c|c|}
\hline Variável & $\begin{array}{c}\text { Total de } \\
\text { participantes } \\
\text { n=772 }\end{array}$ & $\begin{array}{l}\text { Não uso do preservativo } \\
\qquad n=188(\%)\end{array}$ & RP (IC 95\%) & $\mathbf{p}$ \\
\hline \multicolumn{5}{|c|}{ Dados. Sociodemográficos } \\
\hline Idade & & & & $<0,01$ \\
\hline $16-20$ & 139 & $15(10,8)$ & 1 & \\
\hline $21-25$ & 299 & $62(20,7)$ & $1,92(1,13-3,25)$ & \\
\hline $26-30$ & 143 & $40(28,0)$ & $2,59(1,50-4,47)$ & \\
\hline $31-40$ & 119 & $39(32,8)$ & $3,03(1,76-5,22)$ & \\
\hline$\geq 41$ & 72 & $32(44,4)$ & $4,12(2,39-7,09)$ & \\
\hline Sexo & & & & 0,50 \\
\hline Masculino & 382 & $89(23,3)$ & 1 & \\
\hline Feminino & 390 & $99(25,4)$ & $1,09(0,85-1,39)$ & \\
\hline \multicolumn{5}{|l|}{ Raça/cor } \\
\hline Não branco & 555 & $128(23,1)$ & 1 & 0,18 \\
\hline Branco & 217 & $60(27,6)$ & $1,20(0,92-1,56)$ & \\
\hline \multicolumn{5}{|l|}{ Estado civil } \\
\hline Sem companheiro & 523 & $82(15,7)$ & 1 & $<0,01$ \\
\hline Com companheiro & 249 & $106(42,6)$ & $2,71(2,12-3,47)$ & \\
\hline Renda familiar & & & & 0,19 \\
\hline$<2$ salários mínimos & 139 & $28(20,1)$ & 1 & \\
\hline 2-4 salários mínimos & 344 & $80(23,3)$ & $1,15(0,79-1,69)$ & \\
\hline > 5 salários & 289 & $80(27,7)$ & $1,37(0,94-2,00)$ & \\
\hline Religião & & & & 0,37 \\
\hline Protestante & 216 & $60(27,8)$ & 1 & \\
\hline Católico & 311 & $66(21,2)$ & $0,76(0,56-1,03)$ & \\
\hline Outros & 79 & $20(25,3)$ & $0,91(0,59-1,41)$ & \\
\hline Não tem & 166 & $42(25,3)$ & $0,91(0,65-1,28)$ & \\
\hline
\end{tabular}

Fonte: Dados da pesquisa, 2016. Conhecimento sobre IST/HIV/Aids e outros agravos: Atitudes e práticas preventivas entre Universitários. Freitas JLG; Pereira PPS; Moreira KFA; Silva, AD.

$\mathrm{Na}$ análise bivariada apenas as variáveis idade e estado civil apresentaram significância estatística em relação ao desfecho. Os resultados mostram que a razão de prevalência (RP) aumenta em função da idade, sendo que para os participantes acima de 41 anos a razão de prevalência do não uso de preservativos foi 4,12 (IC95\%: 2,39-7,09). Quanto ao estado civil, a RP para o não uso de preservativo entre os participantes com companheiro foi de 2,71 (IC 95\%: 2,12-3,47), indicando que há menor adesão ao uso de preservativos entre os casados e com união estável.

Apenas uma variável foi significativa no que se refere a informações educacionais. Estudantes matriculados em períodos finais do curso ou pós-graduação apresentaram razão de prevalência de não uso de preservativo de 1,44 (IC 95\%: 1,06-1,95) quando comparado aso estudantes nos períodos iniciais do curso. 
Quanto às informações educacionais e de comportamento sexual (Tabela 2), a maioria dos estudantes não eram de cursos da área da saúde $(78,4 \%)$, estavam frequentando períodos intermediários, finais ou pósgraduação $(76,3 \%)$, tinha como principal fonte de informação sobre saúde sexual o tipo pessoal que se refere a amigos, familiares e profissionais de saúde $(68,3 \%)$ e tiveram apenas um parceiro sexual nos últimos 12 meses $(66,1 \%)$.

E em relação ao comportamento sexual, os estudantes com parceiro fixo nos últimos 12 anos tiveram maior probabilidade para o não uso do preservativo nas últimas cinco relações sexuais $(R P=5,28$; IC95\%: $2,77-10,06)$ quando comparado aos que tiveram mais de dois parceiros no período.

Tabela 2 - Características educacionais e comportamento sexual dos universitários e pós-graduandos e não uso de preservativo nas últimas cinco relações sexuais, n=772. Porto Velho, RO, 2016.

\begin{tabular}{|c|c|c|c|c|}
\hline Variável & $\begin{array}{c}\text { Total de } \\
\text { participantes } \\
n=772\end{array}$ & $\begin{array}{l}\text { Não uso do preservativo } \\
\qquad n=188(\%)\end{array}$ & RP (IC 95\%) & $\begin{array}{l}\text { Valor } \\
\text { de } p\end{array}$ \\
\hline \multicolumn{5}{|l|}{ Dados Educacionais } \\
\hline Curso da área da saúde & & & & 0,08 \\
\hline Não & 605 & $156(26,0)$ & 1 & \\
\hline $\operatorname{Sim}$ & 167 & $32(19,2)$ & $0,74(0,53-1,04)$ & \\
\hline Período do curso & & & & 0,05 \\
\hline Iniciante (1-3 sem.) & 276 & $68(21,0)$ & 1 & \\
\hline Intermediário (4-6 sem.) & 274 & $63(23,0)$ & $1,09(0,80-1,50)$ & \\
\hline Término (7-10 sem./pós-graduandos) & 222 & $67(31,2)$ & $1,44(1,06-1,95)$ & \\
\hline \multicolumn{5}{|l|}{ Fontes de informação em saúde } \\
\hline Impessoais & 245 & $55(22,3)$ & 1 & 0,40 \\
\hline Pessoais & 527 & $133(25,3)$ & $1,12(0,85-1,48)$ & \\
\hline \multicolumn{5}{|l|}{ Bloco 3. Comportamento sexual } \\
\hline Parceiros nos últimos 12 meses & & & & $<0,01$ \\
\hline Três ou mais & 146 & $9(6,2)$ & 1 & \\
\hline Dois & 95 & $9(9,5)$ & $1,54(0,63-3,73)$ & \\
\hline Um & 510 & $166(32,5)$ & $5,28(2,77-10,06)$ & \\
\hline Nenhum & 21 & $4(19,1)$ & $3,09(1,04-9,15)$ & \\
\hline
\end{tabular}

Fonte: Dados da pesquisa, 2016. Conhecimento sobre IST/HIV/Aids e outros agravos: Atitudes e práticas preventivas entre Universitários.

Entre os participantes com parceiro único nos últimos 12 meses a razão de prevalência para o não uso de preservativo nas últimas cinco relações sexuais foi 3,84 (IC 95\%: 1,99-7,40). O teste estatístico de HosmerLemeshow foi aplicado para verificar a qualidade de ajuste do modelo final. O valor de $p$ foi 0,1656 e a hipótese nula foi rejeitada, indicando boa qualidade do modelo.

Na Tabela 3, são apresentados os resultados da análise de regressão múltipla de Poisson. No modelo II, a variável período do curso perdeu significância estatística em relação ao desfecho quando ajustada pelas variáveis sociodemográficas (Bloco 1). No modelo III, a condição de ter um único parceiro se manteve associado ao não uso do preservativo mesmo após ajustes pelas variáveis sociodemográficas e educacionais. 
Tabela 3 - Regressão Múltipla de Poison.

\begin{tabular}{|c|c|c|c|}
\hline Variável & $\begin{array}{c}\text { Modelo I } \\
\text { RP (IC 95\%) }\end{array}$ & $\begin{array}{c}\text { Modelo II } \\
\text { RP (IC 95\%) }\end{array}$ & $\begin{array}{c}\text { Modelo III } \\
\text { RP (IC 95\%) }\end{array}$ \\
\hline \multicolumn{4}{|l|}{ Dados Sociodemográficas } \\
\hline \multicolumn{4}{|l|}{ Idade } \\
\hline $21-25$ & $1,62(0,94-2,78)$ & & \\
\hline $26-30$ & $1,91(1,07-3,39)$ & & \\
\hline $31-40$ & $1,81(1,01-3,24)$ & & \\
\hline$>41$ & $2,19(1,21-3,97)$ & & \\
\hline \multicolumn{4}{|l|}{ Raça/cor } \\
\hline Branco & $1,14(0,80-1,47)$ & & \\
\hline \multicolumn{4}{|l|}{ Estado civil } \\
\hline Com companheiro & $1,75(1,29-2,37)$ & & \\
\hline \multicolumn{4}{|l|}{ Renda familiar } \\
\hline 2-4 salários mínimos & $1,09(0,75-1,59)$ & & \\
\hline >5 salários mínimos & $1,25(0,86-1,81)$ & & \\
\hline \multicolumn{4}{|l|}{ Bloco 2. Educacionais } \\
\hline \multicolumn{4}{|l|}{ Curso da área da saúde } \\
\hline Sim & & $0,92(0,65-1,28)$ & \\
\hline \multicolumn{4}{|l|}{ Período do curso } \\
\hline Intermediário (4-6 sem.) & & $1,11(0,82-1,49)$ & \\
\hline Término (7-10 sem. ou pós-graduação) & & $1,24(0,93-1,67)$ & \\
\hline \multicolumn{4}{|l|}{ Bloco 3. Comportamento sexual } \\
\hline \multicolumn{4}{|l|}{ Número de parceiros nos últimos 12 meses } \\
\hline Dois & & & $1,41(0,58-3,42)$ \\
\hline Um & & & $3,84(1,99-7,40)$ \\
\hline Nenhum & & & $2,27(0,76-6,71)$ \\
\hline
\end{tabular}

Fonte: Dados da pesquisa, 2016. Conhecimento sobre IST/HIV/Aids e outros agravos: Atitudes e práticas preventivas entre Universitários.

\section{DISCUSSÃO}

Nesse estudo, o perfil dos jovens universitários de ambos os sexos, se assemelham a de outros grupos jovens no Brasil. Possuem majoritariamente menor que 25 anos, não são casados, possuem renda familiar inferior a cinco salários mínimos e se identificam como praticantes entre as religiões existentes no país como observado em outros estudos (PEREIRA BS et al., 2014; FIRMEZA SNRM et al., 2016).

Foi encontrada uma relação linear entre a idade e o não uso do preservativo, ou seja, quanto maior a idade, menor a adesão ao uso do preservativo nas últimas cinco relações sexuais. Manter relações sexuais sem preservativos é um comportamento observado principalmente nas pessoas maduras, com certa estabilidade financeira, que demonstram corretos conhecimentos de prevenção às IST, mas ainda assim possuem maior dificuldade em fazer uso regular do preservativo nas relações estáveis pela confiança no parceiro fixo, na influência da bebida alcoólica e a perca do prazer (MEDEIROS LG et al., 2014).

Os universitários casados ou com união estável tiveram maior prevalência do não uso do preservativo em relação aos que estavam solteiros, namorando, divorciados ou viúvos. De modo similar, os estudantes que tiveram somente um parceiro sexual nos últimos 12 meses, tiveram maior chance de não usar o preservativo do que aqueles com mais de dois parceiros. Nesses achados, em ambas as situações, observa-se a necessidade de se reconhecer múltiplos aspectos de natureza afetivo-normativa que podem estar servindo de barreiras ao uso regular do preservativo no contexto de relações estáveis (DOURADO I et al., 2015).

A renda familiar não foi estatisticamente significante em relação ao desfecho. Na literatura verifica-se que, independente do estrato socioeconômico, os jovens universitários continuam justificando o não uso do preservativo por variados motivos que vão desde a imprevisibilidade das relações sexuais, pela interferência ou desconforto desse método na obtenção do prazer sexual ou da confiança no parceiro estiveram associadas 
ao uso inconsistente do preservativo ou até por questões próprias de contrariar regras sociais (El BCHERAOUI C etal.,2013; PANOBIANCO MS et al., 2013). Em relação aos aspectos educacionais, a maioria dos participantes estava matriculada em períodos finais de seus cursos ou na pós-graduação. Esses achados indicam que nem sempre a educação nos moldes acadêmicos é capaz de ser traduzida em conhecimentos suficientes para pautar condutas de prevenção às IST na vida pessoal (FIRMEZA SNRM et al., 2016; CASTRO EL et al., 2016; MEDEIROS LG et al., 2014).

Além do ensino formal, há outras fontes de informações sobre a saúde sexual, como por exemplo, os amigos, porém esta fonte pode ter um saber limitado sobre o tema. Nesta pesquisa, a maioria dos estudantes obtinham informações sobre saúde sexual na esfera pessoal - amigos, familiares entre outros. A literatura tem demonstrado que o comportamento sexual dos jovens e o nível de conhecimento destes sobre IST/HIV/Aids tem forte associação com o fato de amigos serem as principais referências (FIRMEZA SNRM et al., 2016).

$\mathrm{O}$ acesso às informações equivocadas agrava-se com o despreparo das famílias, dos profissionais de saúde e outros que tenham contato e influência junto aos jovens, bem como a ausência de ações governamentais. A ausência de informações adequadas pode levar à prática sexual insegura, falta de amadurecimento psicoafetivo, maior vulnerabilidade e riscos aos jovens (CASTRO EL et al., 2016; PANOBIANCO MS et al., 2013).

Ainda que a significância estatística entre o não uso de preservativo e o nível de escolaridade não tenha se mantido após ajustes por outras variáveis no modelo final, foi possível verificar que o maior tempo de permanência na universidade não resultou em práticas sexuais mais seguras já que neste grupo foi encontrada a maior prevalência do não uso do preservativo na análise bivariada. Corroborando com este achado, um estudo feito em Portugal com universitários de várias nacionalidades verificou que apesar dos estudantes saberem que o preservativo é a única forma de prevenção de IST/HIV/Aids, a maioria dos participantes não utilizaram preservativos na sua última relação sexual (GRAVATA A et al., 2016).

Entre os universitários e pós-graduandos participantes deste estudo 75,6\% utilizaram o preservativo em pelo menos uma vez nas últimas cinco relações sexuais. Em um estudo realizado no Sudeste do país, encontrou que $99 \%$ dos graduandos fizeram uso do preservativo em suas relações, mas menos de $20 \%$ deles fizeram uso constante ou tinham conhecimento para tê-lo usado adequadamente. A informação nesta área deve ser a mais exata e completa possível, de forma que o indivíduo possa ter consciência dos riscos aos quais se expõe e que podem também afetar terceiros na sua prática sexual (CASTRO EL et al., 2016).

A literatura destaca que embora os adultos jovens sempre se mostrarem favoráveis à incorporação de métodos preventivos às IST, persistem com atitudes e práticas de sexo inseguro, seja pelo não uso ou o uso descontinuado do preservativo (VALENCIA CP e CARVAJAL EG, 2012; DOURADO I et al, 2015). Embora não tenham sido avaliadas questões sobre o acesso ao preservativo por estudante do presente estudo, chama a atenção o fato de que seus participantes contavam com distribuição regular e gratuita de preservativos no ano do estudo na universidade por meio de ações de extensão já instituídas (UNIVERSIDADE FEDERAL DE RONDÔNIA, 2015).

Sabe-se que a disponibilidade do preservativo no ambiente universitário de forma gratuita é um fator importante na promoção do uso desses insumos. Quando associado a programas de prevenção ao IST essas ações incrementam o nível de conhecimento e a percepção sobre os riscos das práticas sexuais desprotegidas (DOURADO I et al., 2015; FRANCIS DB et al., 2016).

Estudo em uma universidade pública no sudoeste dos Estados Unidos em 2013, apontou que a garantia do preservativo com livre acesso na universidade foi um preditor significativo da intenção genuína dos alunos sexualmente ativos em buscar os dispensadores do insumo. Os resultados indicaram que, durante um curto período de tempo, a distribuição de preservativos foi bem-sucedida por atingir os alunos e oferecer preservativos gratuitos (DELGADO MPT e CRUZ MECS, 2015). A presente investigação traçou alguns aspectos do comportamento sexual de estudantes universitários de uma região Norte do país, relativo ao não uso do preservativo, considerando fatores sociodemográficas, educacionais e comportamentais confrontado com a literatura atual sobre a temática. Outras variáveis não analisadas podem ter influenciado 
significativamente os resultados, a exemplo do uso de álcool e drogas ilícitas, variáveis de reconhecido impactos na motivação do uso (ou não) do preservativo. Em virtude do delineamento transversal da investigação, não foi possível estabelecer relações causa-efeito, portanto os resultados devem ser interpretados com cautela considerando as particularidades do método e da amostra utilizada, já que seus participantes pertenciam a cursos presenciais da única universidade pública do estado. Além disso, admitese a presença de viés de memória dado que a pesquisa utilizou informações autorreferidas do tipo recordatário.

Entretanto, acredita-se que os resultados encontrados possam ser úteis a contextos sociais semelhantes à instituição estudada. Neste caso, verifica-se a necessidade de implementar e/ou reforçar ações de ensino e extensão nesta área, por meio de estratégias educativas que estimulem a discussão sobre a temática e assim possa refletir em uma maior adesão ao preservativo entre os jovens universitários. Esperava-se que os universitários dos períodos iniciais tivessem maior comportamento de risco, quanto ao não uso de preservativos, do que os de períodos finais e pós-graduação, porém o que foi observado foi uma maior prevalência do não uso do preservativo nas últimas cinco relações sexuais entre os estudantes de períodos finais ou pós-graduação. A maior prevalência de não uso de preservativos entre pessoas com mais de 40 anos de idade, remete a existência de falhas na qualidade das informações formais e informais obtidas sobre IST/HIV/Aids ao longo da formação acadêmica e da vida, o que pode estar interferindo na percepção de risco para mudança de atitudes e práticas saudáveis nesse grupo.

\section{CONCLUSÃO}

Nesse estudo, universitários e pós-graduandos, sobretudo aqueles com mais de 40 anos, com parceiros únicos e nos finais de curso estão mais vulneráveis às IST/HIV/Aids já que fazem menos uso do preservativo. È necessário que revejam suas práticas sexuais com conhecimento adequado e atitude de proteção junto aos seus pares e para si mesmo, já que muitos serão formadores de opinião nos diversos locais onde estarão inseridos. Pelo convívio diário e prolongando, cabe à universidade como fórum privilegiado, investir em ações e/ou projetos de extensão na área de saúde sexual e reprodutiva de jovens universitários, a exemplo desta instituição pesquisada.

\section{AGRADECIMENTOS}

Às alunas Alexandra Araújo Santos e Lizabeth Jeffryes Lima Reis Filha, acadêmicas do curso de Enfermagem da Universidade Federal de Rondônia, que contribuíram na fase da coleta de dados.

\section{REFERÊNCIAS}

1. ARRIAGADA EMB, BERTONI JS. Una revisión de los estilos de vida de estudiantes universitarios Iberoamericanos. Cienc Enferm. 2014; 20 (2): 93- 101.

2. PEREIRA BS et al. Fatores associados à infecção pelo HIV/AIDS entre adolescentes e adultos jovens matriculados em Centro de Testagem e Aconselhamento no Estado da Bahia, Brasil. Ciênc Saúde Coletiva. 2014; 19 (3): 747-58.

3. BEZERRA EO et al. Análise da vulnerabilidade sexual de estudantes universitários ao HIV/AIDS. Rev Rene. 2012; 13 (5): 1121-1131.

4. BRASIL. Instituto Nacional de Estudos e Pesquisas Educacionais Anísio Teixeira. Sinopse estatística da educação superior. 2015.

5. BRASIL. Ministério da Saúde. Boletim epidemiológico HIV/AIDS. Secretaria de Vigilância em Saúde. Volume 49, no 53- julho de 2017 a junho de 2018. Brasília: MS; 2018.72p.

6. GRAVATA A et al. Study of the Sociodemographic Factors and Risky Behaviours Associated with the Acquisition of Sexual Transmitted Infections by Foreign Exchange Students in Portugal. Acta Med Port. 2016; 29 (6): 360 366.

7. SALES WB et al. Risky sexual behavior and knowledge of STIS/AIDS among university health students. Rev Enferm Ref. 2016; 4 (10): 19-27. 
8. VALENCIA CP, CARVAJAL, EG. Factores que predisponen, facilitan y refuerzan el uso del preservativo en jóvenes universitarios de Cali, Colombia. Rev Salud Publica. 2012; 14 (5): 810-821.

9. REYNOLDS HW et al. The measurement of condom uses in four countries in East and southern Africa. AIDS end Behavior. 2012; 16 (4): 1044-1053.

10. DOURADO I et al. Revisiting the use of condoms in Brazil. Rev Bras Epidemiol. 2015;18(1): 63-88.

11. EI BCHERAOUI $\mathrm{C}$ et al. Patterns of condom use among students at historically Black colleges and universities: Implications for HIV prevention efforts among college-age young adults. AIDS Care. 2013; 25 (2):186-192.

12. FIRMEZA SNRM et al. Comportamento sexual entre acadêmicos de uma universidade pública. Rev Rene. 2016; 17 (4): 506-511.

13. CASTRO EL et al. O conhecimento e o ensino sobre doenças sexualmente transmissíveis entre universitários. Cien. Saude Colet. 2016; 21 (6): 1975-1984.

14. Borges ALV et al. ERICA: sexual initiation and contraception in Brazilian adolescents. Rev Saude Pub. $2016 ; 50$ (1): $1-11$.

15. MEDEIROS LG et al. Conhecimento e vulnerabilidade de professores Universitários do sexo masculino às doenças sexualmente transmissíveis. Rev Saúde Com. 2014; 10(1): 33-42.

16. PANOBIANCO MS et al. Knowledge concerning HPV among adolescent undergraduate nursing students. Texto Contexto Enferm. 2013; 22 (1): 201-207.

17. UNIVERSIDADE FEDERAL DE RONDÔNIA. Pró-Reitoria de Cultura, Extensão e Assuntos Estudantis da Universidade Federal de Rondônia. 2012.

18. FRANCIS DB et al. Perceptions of a campus-wide condom distribution programme: An exploratory study. Health Educ J. 2016; 75(8): 998-1011.

19. JANEIRO JM et al. Sexual and contraceptives attitudes, the locus of health control and self-esteem among higher education students. Rev Bras Promo Saude. 2013; 26 (4): 505-512.

20. DELGADO MPT, Cruz MECS. Efectividad de un programa educativo sobre VIH/Sida aplicado a universitarios dentro del enfoque promocional. Cuid Salud. 2015; 2 (1):125-136. 